\title{
High serum N-terminal propeptide of procollagen type III concentration is associated with liver diseases
}

\author{
Monika Gudowska ${ }^{1}$ Ewa Gruszewska ${ }^{1}$, Anatol Panasiuk², Bogdan Cylwik ${ }^{3}$, Magdalena Swiderska $^{2}$,

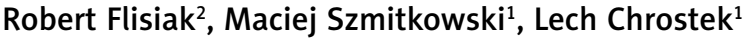 \\ ${ }^{1}$ Department of Biochemical Diagnostics, Medical University of Bialystok, Bialystok, Poland \\ ${ }^{2}$ Department of Infectious Diseases and Hepatology, Medical University of Bialystok, Bialystok, Poland \\ ${ }^{3}$ Department of Paediatric Laboratory Diagnostics, Medical University of Bialystok, Bialystok, Poland
}

Gastroenterology Rev 2017; 12 (3): 203-207

DOI: https://doi.org/10.5114/pg.2017.70474

Key words: N-terminal propeptide of procollagen type III, liver cirrhosis, toxic hepatitis, non-invasive markers.

Address for correspondence: Prof. Lech Chrostek, Department of Biochemical Diagnostics, Medical University of Bialystok, 15 A Waszyngtona St, 15-269 Bialystok, Poland, phone/fax: +48 8574685 85, e-mail: chrostek@umb.edu.pl

\begin{abstract}
Introduction: N-terminal propeptide of procollagen type III (PIIINP) is generated during the synthesis of type III collagen. PIIINP can be measured in the serum as an indicator of liver fibrosis and cirrhosis.

Aim: To evaluate the effect of liver diseases of different aetiologies and clinical severity of liver cirrhosis on the serum level of PIIINP.

Material and methods: Patients with alcoholic cirrhosis (AC) - 63 subjects, non-alcoholic cirrhosis (NAC) - 31 and toxic hepatitis (HT) - 33 were studied. Cirrhotic patients were classified according to the Child-Pugh scale. The samples were analysed using the ELISA method.

Results: The level of PIIINP was significantly higher in patients with alcoholic cirrhosis, non-alcoholic cirrhosis, and toxic hepatitis in comparison to the control group. There were no significant differences in the serum PIIINP levels between liver diseases and according to the severity of liver cirrhosis. PIIINP has the highest diagnostic power for the diagnosis of toxic hepatitis. The highest sensitivity was reached in alcoholic cirrhosis, but other diagnostic values (specificity, positive predictive value (PPV), negative predictive value (NPV), diagnostic accuracy (ACC)) in alcoholic cirrhosis were lower than that in toxic hepatitis. In the diagnosis of non-alcoholic cirrhosis PIIINP has low sensitivity, specificity, PPV, NPV, and ACC.

Conclusions: The serum PIIINP shows the alterations in liver diseases in comparison to healthy controls, but not between diseases. Taking the above into account we can suggest that PIIINP may be a useful test for the detection of liver diseases.
\end{abstract}

\section{Introduction}

Liver biopsy is recommended as the "gold standard" for the detection of liver diseases. However, liver biopsy is an invasive procedure with a risk of complications, and its sensitivity in diagnosing of cirrhosis is not absolute and sampling error is very common $[1,2]$. Therefore, the laboratory tests (non-invasive) would be useful in the diagnosis of liver fibrosis and cirrhosis. The process of liver fibrosis is assumed to be caused by the excess production of extracellular matrix (ECM) in hepatic stellate cells (HSCs) [3]. The increase of ECM deposited fragments (approximately six times more) in the Disse's space leads to liver injury $[4,5]$. The largest component of the ECM is collagen [6]. In the liver type III collagen mainly occurs $[7,8]$. During the synthesis of type III collagen, the N-terminal propeptide of procollagen type III (PIIINP) is detached from procollagen type III [9], so the fibrogenesis results in release of ECM fragments into the blood [9]. Hence the quantity of propeptide of procollagen type III can be a direct indicator of collagen synthesis and its deposition in the extracellular space [9]. The most common causative agents of fibrosis are: alcoholic liver disease, viral hepatitis B and C, iron or copper overload, and autoimmune states [5]. The increased amount of propeptide of type III procollagen may indicate the transformation of normal liver tissue into connective tissue [5], but PIIINP is not a liver-specific marker. Increased concentrations of PIIINP were also observed in other pathological conditions associated with abnormal production of collagen, such as conges- 
tive heart failure, hypertension, and coronary heart disease. It is probably caused by ongoing inflammation [7].

\section{Aim}

The aim of this study was to evaluate the effect of liver diseases of different aetiologies (alcoholic and non-alcoholic) and clinical severity of liver cirrhosis on the serum level of PIIINP.

\section{Material and methods}

The tested group consisted of 127 consecutive patients admitted to the Department of Infectious Diseases and Hepatology (Medical University of Bialystok). The tested patients were both males $(n=85)$ and females $(n=42)$ of ages between 24 and 88 years. They were divided into three subgroups according to the clinical diagnosis of disease: alcoholic cirrhosis (AC) - $63 \mathrm{pa}$ tients, non-alcoholic cirrhosis (NAC) - 31 patients, and toxic hepatitis $(\mathrm{HT})-33$ patients. The diagnosis was based on the signs, symptoms, physical exams, and laboratory liver panel: platelet count (PLT), mean corpuscular volume (MCV), normalised ratio value (INR), aspartate aminotransferase (AST), alanine aminotransferase (ALT), $\gamma$-glutamyl transferase (GGT), albumin, and bilirubin. Data on age, gender, and levels of serum liver enzymes are presented in Table I.

\section{Blood specimen}

Venous blood samples were collected from every patient by peripheral vein puncture. The sera were separated by centrifugation at $1500 \times \mathrm{g}$ for $10 \mathrm{~min}$ at room temperature and stored at $-86^{\circ} \mathrm{C}$ until assayed. PIIINP was measured with enzyme-linked immunosorbent assay - ELISA (EIAab, China) according to the manufacturer's protocols. Duplicate samples were assessed for each patient. The ALT, AST, GGT, and bilirubin were measured on an ARCHITECT ci8200 (Abbott Laboratories) according to the spectrophotometric method and using Abbott reagents. The PLT count and MCV were determined on a Sysmex XS800i (Sysmex Corporation), and INR on an STA Compact Max analyser (Diagnostica Stago, Inc.) by the viscometric method.

\section{Control group}

The control group consisted of 30 healthy volunteers (16 men and 14 women). All subject individuals (healthy and sick) gave written informed consent to participate in our study. The study was approved by the Bioethical Committee at the Medical University in Bialystok, Poland.

\section{Statistical analysis}

Results were expressed as mean and standard deviation (SD). The significance of differences between groups (tested and control) was estimated by Mann-Whitney $U$ test. The analysis of PIIINP according to the aetiology and severity of liver cirrhosis were performed by the ANOVA rank Kruskal-Wallis test. The differences were considered statistically significant at $p<0.05$.

\section{Results}

The mean level of PIIINP was significantly higher in patients with alcoholic cirrhosis $(8.031 \pm 2.494 \mathrm{ng} /$ $\mathrm{ml})$, non-alcoholic cirrhosis $(7.557 \pm 3.306 \mathrm{ng} / \mathrm{ml})$, and toxic hepatitis $(8.509 \pm 2.476 \mathrm{ng} / \mathrm{ml})$ in comparison to the control group $(5.200 \pm 0.861 \mathrm{ng} / \mathrm{ml})(p<0.001$, $p=0.001, p<0.001$, respectively) (Figure 1), but there were no significant differences in the serum PIIINP levels between liver diseases (ANOVA: $H=1.558, p=$ 0.459). The values of PIIINP in Child-Pugh class A, B,

Table I. Changes of blood liver parameters

\begin{tabular}{|c|c|c|c|c|}
\hline Parameter & $C(n=20)$ & $\mathrm{AC}(n=63)$ & NAC $(n=31)$ & $\mathrm{HT}(n=33)$ \\
\hline $\operatorname{PLT}\left[\times 10^{3} / \mu \mathrm{l}\right]$ & $235.35 \pm 51.37$ & $125.31 \pm 104.40$ & $101.09 \pm 49.18$ & $186.48 \pm 85.37$ \\
\hline$M C V[f l]$ & $87.06 \pm 4.49$ & $97.02 \pm 8.84$ & $90.10 \pm 7.77$ & $96.31 \pm 6.85$ \\
\hline INR & $0.93 \pm 0.04$ & $1.42 \pm 0.39$ & $1.15 \pm 0.20$ & $1.19 \pm 0.53$ \\
\hline AST [IU/I] & $23.25 \pm 5.31$ & $103.93 \pm 112.78$ & $75.43 \pm 59.78$ & $149.13 \pm 126.13$ \\
\hline $\mathrm{ALT}[\mathrm{IU} / \mathrm{I}]$ & $17.65 \pm 8.29$ & $44.76 \pm 47.91$ & $58.63 \pm 70.09$ & $177.12 \pm 191.41$ \\
\hline GGT [IU/I] & $23.30 \pm 7.33$ & $390.97 \pm 505.18$ & $100.32 \pm 83.33$ & $914.25 \pm 868.51$ \\
\hline Total bilirubin $[\mu \mathrm{M} / \mathrm{l}]$ & $17.27 \pm 7.52$ & $110.12 \pm 128.93$ & $21.55 \pm 12.83$ & $102.09 \pm 137.14$ \\
\hline Albumin [g/dl] & $3.98 \pm 1.12$ & $3.06 \pm 0.65$ & $3.43 \pm 0.60$ & $3.58 \pm 0.57$ \\
\hline
\end{tabular}

Data are mean \pm standard deviation. C - controls, AC - alcoholic cirrhosis, NAC - non-alcoholic cirrhosis, HT-toxic hepatitis, PLT - platelet count, $M C V$ - mean corpuscular volume, INR - international normalised ratio, AST - aspartate aminotransferase, ALT - alanine aminotransferase, GGT- $\gamma$-glutamyl transferase. 

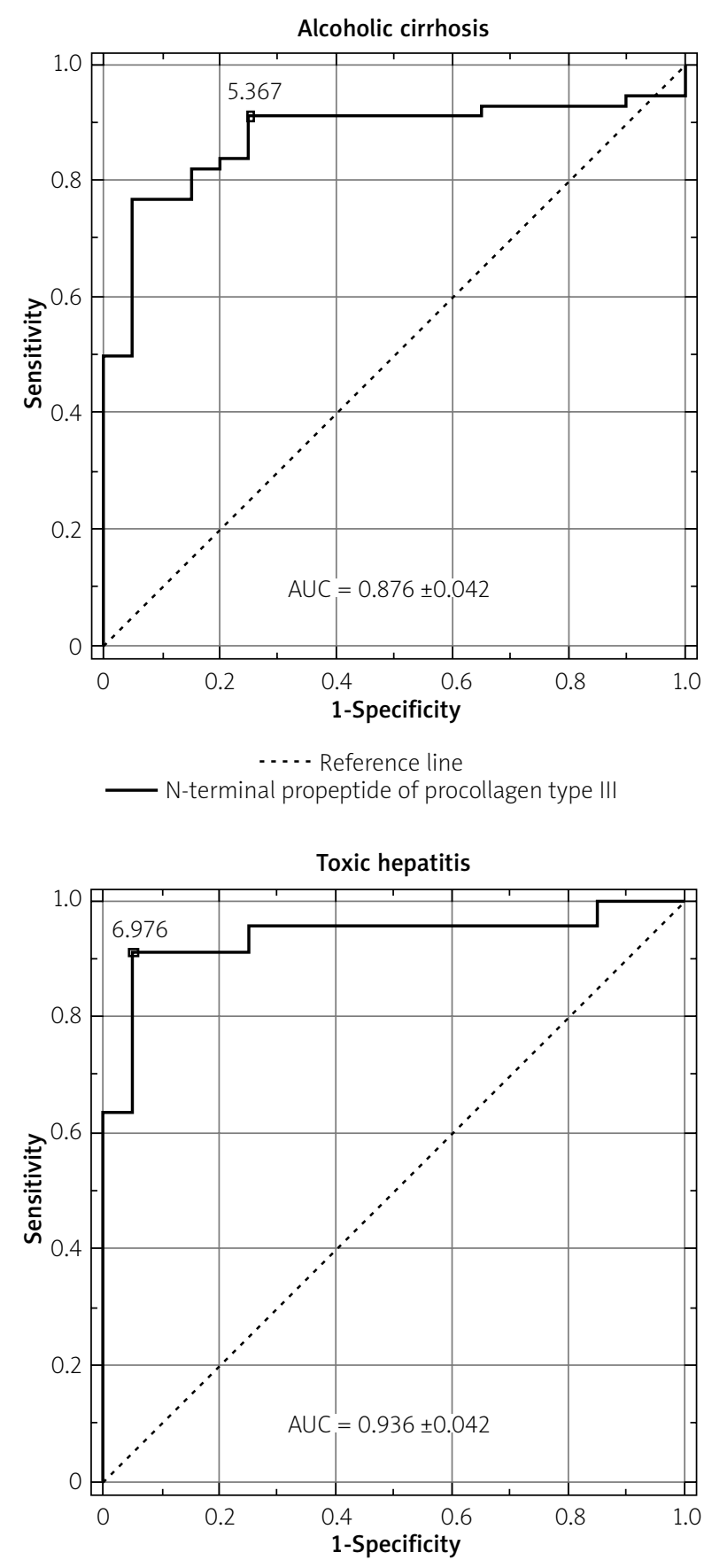

....- Reference line

$\mathrm{N}$-terminal propeptide of procollagen type III

and $C$ were similar $(8.219 \pm 3.609,8.222 \pm 2.500,7.306$ \pm 2.108 , respectively). The analysis of variance revealed that severity of liver cirrhosis did not affect the value of PIIINP $(H=5.926, p=0.051)$.

The ROC curves of serum PIIINP in liver diseases are presented in Figure 2. PIIINP has the highest diagnostic power for the diagnosis of toxic hepatitis at the cut-off point of $6.976 \mathrm{ng} / \mathrm{ml}$. The diagnostic sensitivity of PIIINP

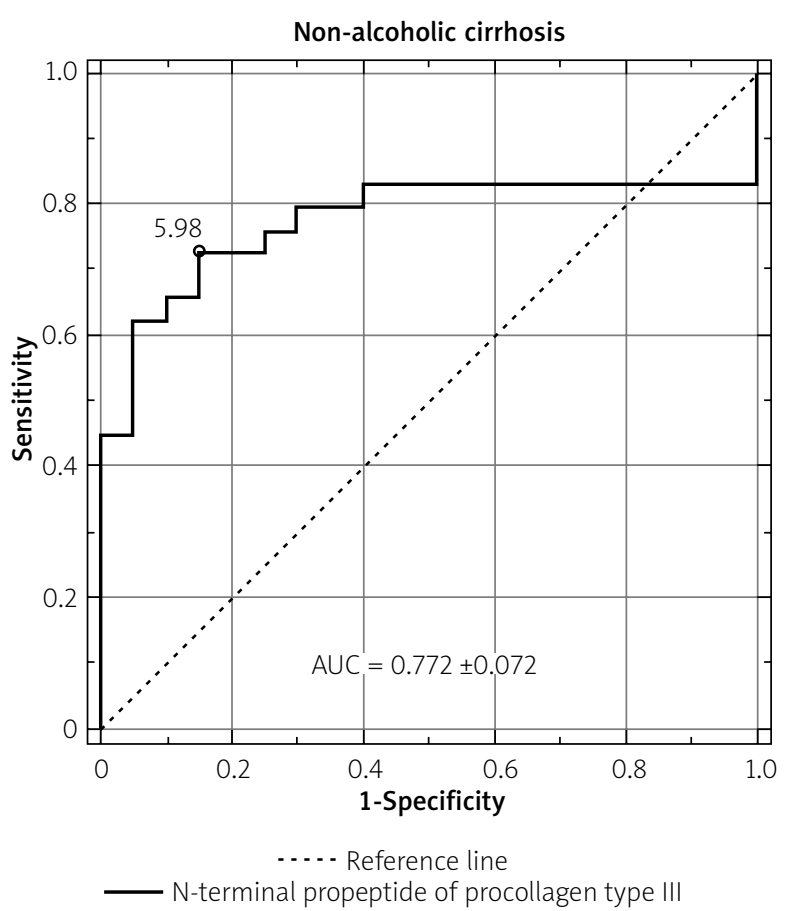

Figure 1. The ROC curves of N-terminal propeptide of procollagen type III in liver diseases

in toxic hepatitis is equal to $90.9 \%$, specificity - $95 \%$, positive predictive value (PPV) $-95.2 \%$, negative predictive value (NPV) $-90.5 \%$, and diagnostic accuracy (ACC) - 92.9\%. PIIINP has the highest sensitivity (91.1\%) in alcoholic cirrhosis at the cut-off point of $5.367 \mathrm{ng} / \mathrm{ml}$, but other diagnostic values (specificity - 75\%, PPV $91.1 \%$, NPV - 75\%, ACC - 86.8\%) in alcoholic cirrhosis were lower in comparison with toxic hepatitis. In diag- 


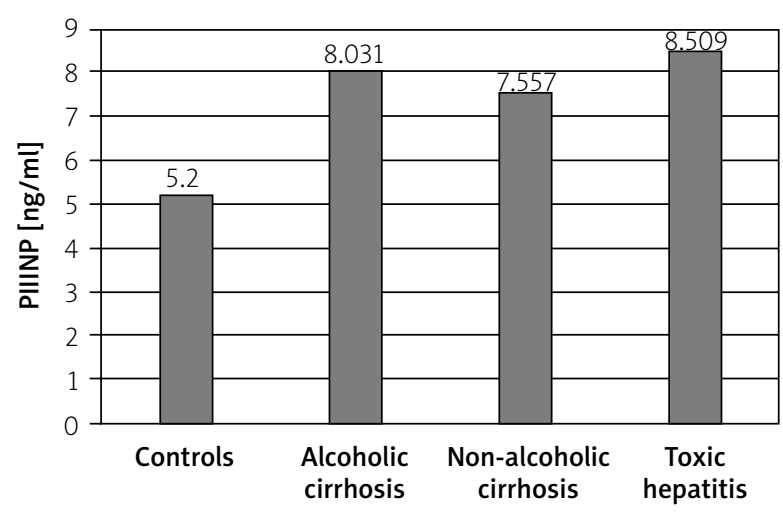

Figure 2. The levels of serum N-terminal propeptide of procollagen (PIIINP) type III in liver diseases

nosis of non-alcoholic cirrhosis PIIINP has low sensitivity (72.\%), specificity (85\%), PPV (87.5\%), NPV (68\%), and ACC (77.6\%).

\section{Discussion}

Studies based on PIIINP as a liver damage indicator suggest that tissues with high fibrogenic activity or non-mature fibrotic tissue consist mostly of type III collagen, whereas in mature fibrotic tissue the levels of collagen type III are lower. Also, tissues undergoing healing have increased levels of PIIINP [4]. So it is problematic to distinguish mild stages of liver fibrosis from the process of healing and regeneration. Therefore, we tried to examine if the level of serum PIIINP reflects the liver injury and process of fibrogenesis.

In this study we have shown that the mean serum level of procollagen III N-terminal propeptide is elevated in patients with liver diseases. Our findings are similar to the results reported by Nojgaard et al., who determined samples from patients using radioimmunoassay (RIA) method. They revealed an increased expression of PIIINP in patients suffering from different alcoholic liver diseases compared to controls [4]. Additionally, Parsian et al. showed that the mean serum PIIINP levels are higher in HBV and immune hepatitis than that in the control group [10]. It is probably the result of the increased hepatic synthesis and turnover of ECM [11], and also a diminishing hepatic clearance [12]. On the other hand, we observed that PIIINP has the highest diagnostic power for the diagnosis of toxic hepatitis (good sensitivity, specificity, PPV, and NPV). Therefore, we can speculate that PIIINP is a better indicator of inflammation than cirrhosis [13].

In this study we did not find any differences in PIIINP serum levels between alcoholic cirrhosis, non-alcoholic cirrhosis, and toxic hepatitis. These findings are different to those obtained in the study of Nojgaard et al., who observed higher levels of serum PIIINP in non-cirrhotic patients with alcoholic hepatitis than non-cirrhotic patients with hepatitis [6]. Collazos and Diaz reported the association between PIIINP and portal hypertension, which was probably due to the fact that the most patients with this disease has also cirrhosis [11]. Similar results were obtained by Jansen et al., who observed the correlation of PIIINP in HIV-HCV co-infected patients with hepatic venous pressure gradient [14]. They also reported that PIIINP level is associated with the stage of fibrosis stratified by FIB-4 score [14]. Also, Nielsen et al. revealed that PIIINP level is higher in patients with chronic hepatitis $C$ in higher stage of fibrosis (Ishak stage 4 compared to stage 2 ), and it is a good indicator to differentiate advanced from mild fibrosis [15]. The one possible explanation for increased PIIINP levels in advanced fibrosis is the fact that ECM remodelling occurs as an attempt to repair damaged tissue in response to liver injury. If the liver damage is extensive the synthesis of ECM components, and collagens, is greater. However, there are no studies comparing PIIINP concentration with severity of liver damage expressed in Child-Pugh scale. Our study revealed that the values of PIIINP are similar independently of the severity of liver cirrhosis.

\section{Conclusions}

We can state that the levels of the PIIINP were elevated in the sera of patients with liver diseases compared to healthy patients. However, PIIINP levels were similar in liver diseases of different aetiologies and according to the severity of liver cirrhosis. Therefore, we can suggest that PIIINP may be a useful test for liver diseases detection, but it does not reflect the stage of liver damage.

\section{Conflict of interest}

The authors declare no conflict of interest.

\section{References}

1. Gressner OA, Weiskirchen R, Gressner AM. Biomarkers of liver fibrosis: clinical translation of molecular pathogenesis or based on liver-dependent malfunction tests. Clin Chim Acta 2007; 381: 107-13.

2. Muiznieks LD, Keeley FW. Molecular assembly and mechanical properties of the extracellular matrix: a fibrous protein perspective. Biochim Biophys Acta 2013; 1832: 866-75.

3. Liu X, Wu H, Byrne M, et al. Type III collagen is crucial for collagen I fibrillogenesis and for normal cardiovascular development. Proc Natl Acad Sci U S A 1997; 94: 1852-6.

4. Bataller R, Brenner DA. Liver fibrosis. J Clin Invest 2005; 115 : 209-18.

5. Sarah D, Berry SD, Vasan S, et al. Procollagen type III N-terminal peptide (P3NP) and lean mass: a cross-sectional study. J Fraility Aging 2013; 2: 129-34. 
6. Nojgaard C, Johansen JS, Christensen E, et al. Serum levels of YKL-40 and PIIINP as prognostic markers in patients with alcoholic liver disease. J Hepatol 2003; 39: 179-86.

7. Baranova A, Lal P, Birerdinc A, et al. Non-Invasive markers for hepatic fibrosis. BMC Gastroenterol 2011; 11: 91-6.

8. Colli A, Colucci A, Paggi S, et al. Accuracy of a predictive model for severe hepatic fibrosis or cirrhosis in chronic hepatitis $C$. World J Gastroenterol 2005; 11: 7318-22.

9. Cross TJ, Calvaruso V, Maimone $\mathrm{S}$, et al. Prospective comparison of Fibroscan, King's score and liver biopsy for the assessment of cirrhosis in chronic hepatitis $C$ infection. J Viral Hepat 2010; 17: 546-54.

10. Parsian H, Nouri M, Rahimipour A, et al. Comparison of five liver fibrosis indexes with serum levels of laminin and $\mathrm{N}$ terminal peptide of procollagen type III in chronic hepatitis patients. In: Liver Biopsy. Intech 2011; 343-60.

11. Collazos J, Diaz F. Role of measurement of serum procollagen III N-terminal peptide in the evaluation of liver diseases. Clin Chim Acta 1994; 227: 37-43.

12. Jarcuska P, Janicko $M$, Veseliny $E$, et al. Circulating markers of liver fibrosis progression. Clin Chim Acta 2010; 411: 1009-17.

13. Smedsr B, Perotoft $H$, Gustsfson $S$, et al. Scavenger functions of the liver endothelial cell. Biochem J 1990; 266: 313-27.

14. Jansen C, Leeming DJ, Mandorfer M, et al. PRO-C3-levels in patients with HIV/HCV-co-Infection reflect fibrosis stage and degree of portal hypertension. PLoS One 2014; 9: e108544.

15. Nielsen MJ, Veidal SS, Karsdal MA, et al. Plasma pro-C3 (N-terminal type III collagen propeptide) predicts fibrosis progression in patients with chronic hepatitis C. Liver Int 2015; 35: 429-37.

Received: 19.01.2016

Accepted: 10.05.2016 